\section{Reducing Individual Heat Stress Through Path Planning}

\author{
Joachim Rußig' and Julian Bruns² \\ 'FZl Forschungszentrum Informatik, Germany \\ ${ }^{2}$ Karlsruhe Institute of Technology, Germany
}

GI_Forum 2017, Issue 1

Page: 327 - 340

Full Paper

Corresponding Author: joachim.russig@alumni.kit.edu

DOI: 10.1553/giscience2017_01_s327

\begin{abstract}
Heat stress is a serious risk, which affects groups such as the elderly or patients with chronic diseases in particular, and is especially pronounced in cities. The ageing of society, progressive urbanization and climate change are increasing the risk of people being affected by heat stress. One way to reduce the risk is to adapt everyday behaviour. To encourage and support such a change of behaviour, we propose a two-step approach. The first step is a route planner for pedestrians which can find a route with minimal heat exposure. The second step is a tool that helps the user to select the time of day with minimal heat exposure to venture outside. The route planner is then used to calculate the heat stress and present the optimal route at that point in time. We evaluate our approach for the city of Karlsruhe. Our results show that the combined approach, as well as its individual steps, can reduce heat exposure and therefore the heat stress for typical daily tasks in a European city.
\end{abstract}

\title{
Keywords:
}

heat stress, time dependent routing, decision support, urban vulnerability

\section{Introduction}

Heat is an important factor for human health and comfort. High temperatures not only lead to discomfort but also have serious negative effects on health as well as on the ability to work.

In numerous studies, an increase in both mortality and morbidity has been associated with a high ambient temperature (Basu, 2009). The most well-known example in recent history is the 2003 heat wave in Europe. Certain groups, such as older people or people with health problems, are especially vulnerable to heat stress (Hübler, Klepper, \& Peterson, 2007). For patients with multiple sclerosis, for example, an increased body temperature can lead to a worsening of their symptoms (Davis, Wilson, White, \& Frohman, 2010). The time that someone must be exposed to heat before it has an effect on them is dependent on factors 
like the ability to adapt to high temperature as well as the activities performed (Hübler, Klepper, \& Peterson, 2007).

Changes such as the ageing of society, increasing urbanization and climate change are making adaptation to the dangers of heat stress more and more important. Because of the rising numbers of people moving into cities, the urban heat island effect (UHI) is set to become increasingly significant in the future. The UHI effect states that an urban area is significantly warmer than surrounding rural areas (Prashad, 2014). The causes of the effect are, especially, the kind of land use and land cover in urban areas, including lower albedo, higher heat capacity and limited green spaces, as well as significant anthropogenic heat releases due to industrial operations or automobile use (Richter, 2015; Oke, 1982). Due to the UHI effect, people living in urban areas are exposed to high ambient temperatures more frequently and for longer periods. They are therefore at greater risk of being affected by the dangers of heat stress.

Individuals can reduce their heat stress by adapting their everyday behaviour. In a typical European city, most typical activities are within walking distance. These can range from going to a grocery store to visiting a doctor. While these activities cannot be omitted, it is possible to use different routes or change the time when they are conducted. In doing so, one can easily reduce the heat stress without a negative impact on the quality of life.

In this paper, we use this reasoning in a two-step approach to help individuals reduce their heat stress. We apply a routing algorithm to compute the optimal path with regard to heat stress. This algorithm is then used to determine the optimal point in time to conduct typical everyday activities. We devised this method for the context of European and European-style cities and evaluated it in the city of Karlsruhe as a representative of this class of cities.

\section{Related Work}

\section{Heat Stress}

The impact of heat on the human body has long been a subject of study. Thermal comfort plays a key role in descriptions of which climatic conditions are considered comfortable.

Staiger, Bucher and Jendritzky (1997) state that only a complete heat budget model of the human body is sufficient to make any reliable statement regarding the influence of heat on the body. The heat budget model of the human body is a simplified model that describes how the internal heat production of the human organism must be balanced with the environment by heat exchange, for instance through skin or respiration (Staiger, Laschewski, \& Grätz, 2012). Some well-known indices that consider a complete human heat budget model are (1) Steadman's heat index (Steadman, 1979a; 1979b), (2) the predicted mean vote (Fanger, 1973), (3) the perceived temperature (Staiger, Bucher, \& Jendritzky, 1997; Jendritzky, Staiger, Bucher, Graetz, \& Laschewski, 2000), and (4) the universal thermal climate index UTCI (Jendritzky, et al., 2010). 
For all these indices, the following meteorological parameters are important: (1) air temperature, (2) water vapour pressure, (3) wind velocity, and (4) mean radiant temperature (Jendritzky, et al., 2010).

Based on the availability of data, in this paper we will use Steadman's heat index (Steadman, 1979a) and, as a simple comparison measure, the air temperature. Steadman's heat index is a single measure for the combined effect of air temperature and humidity (sultriness) and can be used as a measure for heat stress danger (Stull, 2011). The heat index describes how the combination of dry-bulb temperature and vapour pressure feels to a typical human. For the heat index, a typical adult human of either sex with a height of $1.7 \mathrm{~m}$ and a weight of $67 \mathrm{~kg}$, who walks outdoors at a speed of $5 \mathrm{~km} / \mathrm{h}(1.4 \mathrm{~m} / \mathrm{s})$ is considered (Steadman, 1979a).

\section{Health-Optimal Pedestrian Routing}

Several research projects have considered environmental factors for pedestrian routing in the past, with the goal of finding routes which are healthier. For instance, Sharker, Karimi and Zgibor (2012) propose a method to find a health-optimal route, considering environmental factors that include the complexity of the walking trail and the weather. A method to find a route with minimal pollution exposure has been proposed by Hasenfratz (2015).

The NaviComf framework for pedestrian routing proposed by Dang et al. (2013) improves comfort by considering environmental factors which vary over time. Their framework uses a multi-factor cost model for the evaluation of the route and enables the consideration of heterogeneous environmental information from multi-modal sensors. To find an optimal route, Dang et al. (2013) propose three different algorithms - a bounded depth-first search algorithm, an adjustable dynamic planning algorithm, and a heuristic particle planning algorithm. As a sample application, Dang et al. implemented a routing application for thermal comfort navigation. The meteorological data used for this sample application were collected using a network of 40 micro-climate sensor nodes which detected air temperature and relative humidity.

In contrast to the existing work, we contribute an approach which does not rely on extensive sensor networks. We achieve this by combining remote sensing data with fixed weather stations and the use of a static routing algorithm.

\section{Minimize Heat Exposure}

In this work, we reduce the heat stress of people in their everyday life. This is achieved by (1) finding a walking route with minimal heat exposure, and (2) using this route to find the optimal time to start the route. 


\section{Finding a Route with Minimal Heat Exposure}

\section{Modelling as a Time-Dependent Routing Problem}

Finding a route with minimal heat exposure can be modelled as a time-dependent routing problem, where the edge weighting function is not static and may, rather, vary over time. Consequently, many speed-up techniques developed for static routing problems like bidirectional search cannot simply be applied (Delling, Sanders, Schultes, \& Wagner, 2009).

Below, we represent the road network as undirected graph $G=\left(V, E, w_{d}, w_{h}\right)$, where $V$ is the set of vertices or nodes (e.g. junctions) and $\mathrm{E} \subseteq \mathrm{V} \times \mathrm{V}$ is the set of edges (e.g. road segments) which each connect a pair of nodes. $\mathrm{w}_{\mathrm{d}}: \mathrm{E} \rightarrow \mathbb{R}_{\geq 0}$ and $\mathrm{w}_{\mathrm{h}}: \mathrm{E} \times \mathrm{T} \rightarrow \mathbb{R}_{\geq 0}$ are two edge weighting functions, at which:

- $\quad \mathrm{w}_{\mathrm{d}}(\mathrm{e})$ is the length of the edge e and

- $\quad w_{h}(e, t)$ is the heat exposure of edge e at time $t$.

Hereafter, a path $\mathrm{p}$ from node $\mathrm{v}_{0}$ to node $\mathrm{v}_{\mathrm{k}}$ starting a time $\mathrm{t}_{0}$ is given as a sequence of edge-time pairs $\left(\left(\mathrm{e}_{\mathrm{v}_{0} \mathrm{v}_{1}}, \mathrm{t}_{0}\right),\left(\mathrm{e}_{\mathrm{v}_{1} \mathrm{v}_{2}}, \mathrm{t}_{1}\right), \ldots,\left(\mathrm{e}_{\mathrm{v}_{\mathrm{k}-1} \mathrm{v}_{\mathrm{k}}}, \mathrm{t}_{\mathrm{k}-1}\right)\right)$, where $\mathrm{t}_{\mathrm{i}}$ is the time at which node $v_{i}$ is left. The weight of an edge is fixed at the time when traversing the edge is started, the so-called frozen link model (Orda \& Rom, 1990). Time $t_{i}$ can be computed as follows: $t_{i}:=t_{i-1}+t_{w a l k}\left(e_{v_{i-1}, v_{i}}\right)$ where $t_{w a l k}\left(e_{v_{i-1}, v_{i}}\right)$ is the time needed by a pedestrian to traverse the edge $e_{v_{i-1}, v_{i}}$. The starting time $t_{0}$ is either given or set to 0 .

To compute the weight of a path $\mathrm{w}_{\mathrm{h}}(\mathrm{p})$, the following formula can be applied:

$$
w_{h}(p):=\sum_{(e, t) \in p} w_{h}(e, t)
$$

This means that one looks for the path $\mathrm{p}^{*}$ from a node $\mathrm{v}$ to a node $\mathrm{u}$ that has the minimum weight of all possible paths from $v$ to $u$. Below, we use $w_{h}(p, t)$ to denote the weight of path $\mathrm{p}$ starting at time $\mathrm{t}$.

The time-dependent routing problem is $\mathcal{N} \mathcal{P}$-hard if waiting on a node is not permitted and if the FIFO (first in, first out) property is not fulfilled (Orda \& Rom, 1990). A weighting function $\mathrm{w}$ fulfils the FIFO property if the change of the edge weight decreases no faster than the change in the actual time $t$ increases (Kaufman \& Smith, 1993).

Usually, one cannot assume that $\mathrm{w}_{\mathrm{h}}$ fulfils the FIFO property, because the function depends in most instances on the air temperature. In general, the air temperature may decrease more than the actual time increases. Since most people are not willing to wait at a node, finding a route with minimal heat exposure is $\mathcal{N} \mathcal{P}$-hard. Hereafter the edge weighting is frozen at the starting time $\mathrm{t}_{0}$ so that we have a static route-planning problem and classic algorithms like Dijkstra's algorithm (Dijkstra, 1959) can be applied. 


\section{The Edge Weighting Function}

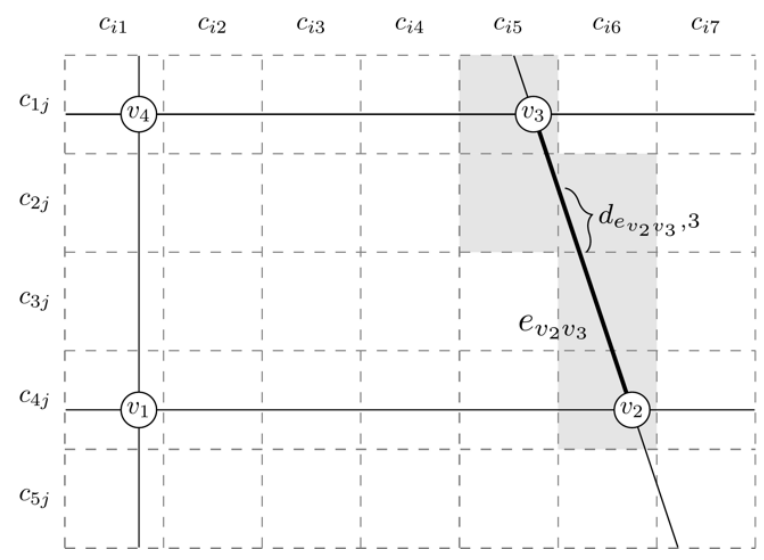

Figure 1: An example for the mapping of the path of a raster file to the edge of a graph network. The values of the raster cells are weighted with the length of the intersection and then accumulated.

To find a route with minimal heat exposure, it is key to define the edge weighting function appropriately. It is not sufficient to take only the actual thermal comfort into account; one must consider the length of time that a person is exposed to heat as well. Below, we assume that the time of exposure is proportional to the length of the edge $\mathrm{w}_{\mathrm{d}}$ (following Hasenfratz, 2015). Based on this assumption, we define the edge weighting function $\mathbf{w}_{\mathrm{h}}$ as follows:

$$
w_{h}(e, t):=\sum_{c \in \text { Intersec }(e)} d_{c} \cdot h_{c}(t)
$$

Here, the thermal comfort values (such as air temperature or heat index) are represented as a time-dependent raster $\mathrm{H}(\mathrm{t})=\left(\mathrm{h}_{\mathrm{ij}}(\mathrm{t})\right)$, where $\mathrm{h}_{\mathrm{ij}}(\mathrm{t})$ denotes the thermal comfort value in raster cell $c_{i j}$ at time $t \in T$. In formula (2) above, Intersec(e) is the set of raster cells intersected by the edge $\mathrm{e}$, and $\mathrm{d}_{\mathrm{c}}$ is the length of the intersection of $\mathrm{e}$ with raster cell $\mathrm{c}$ (see example in Figure 1). That is, to obtain the edge weight, the value of each intersected raster cell is weighted with the length of the intersection and then accumulated.

Using our thermal comfort measures, we get the following edge weighting functions:

$$
\mathrm{w}_{\mathrm{T}_{\mathrm{a}}}(\mathrm{e}, \mathrm{t})=\sum_{\mathrm{c} \in \operatorname{Intersec}(\mathrm{e})} \mathrm{d}_{\mathrm{c}} \cdot \mathrm{T}_{\mathrm{a}}(\mathrm{t}, \mathrm{c})
$$

for the air temperature, and for the heat index we obtain:

$$
\mathrm{w}_{\mathrm{HI}}(\mathrm{e}, \mathrm{t})=\sum_{\mathrm{c} \in \operatorname{Intersec}(\mathrm{e})} \mathrm{d}_{\mathrm{c}} \cdot \mathrm{T}_{\mathrm{HI}}\left(\mathrm{T}_{\mathrm{a}}(\mathrm{t}, \mathrm{c}), \mathrm{RH}(\mathrm{t})\right)
$$


Here, $\mathrm{T}_{\mathrm{a}}(\mathrm{t}, \mathrm{c})$ is the air temperature in raster cell $\mathrm{c}$ at time $\mathrm{t} \in \mathrm{T}, \mathrm{RH}(\mathrm{t})$ is the relative humidity at time $t$, and $\mathrm{T}_{\mathrm{HI}}$ is Steadman's heat index. How we obtained these values is described in section 0 below.

\section{Finding the Optimal Point in Time}

In addition to the optimal route, the appropriate time for doing everyday tasks (e.g. going shopping at a supermarket) can decrease the individual's heat stress significantly. This follows from the fact that heat exposure is normally highest in the middle of the day and lower in the morning or evening.

To give recommendations for a point in time with minimal heat exposure, we use a threestep procedure:

1. Perform a local search originating from a given starting point $\mathbf{s}$ (e.g. address) to find all locations $\mathrm{L}$ that fulfil a certain search criterion (e.g. is a supermarket) within a specified radius $r$ (e.g. $500 \mathrm{~m}$ ).

2. For each location $\ell \in \mathrm{L}$ identified in step 1 , determine the point in time $\mathrm{t}^{*}$ with the lowest heat exposure.

3. Create a ranking of the locations in $\mathrm{L}$ based on the minimum heat exposure found in step 2, ranking the location with the lowest heat exposure as 1 .

As steps 1 and 3 have well-known solutions, we focus on step 2 .

\section{Modelling as an Optimization Problem}

In order to find the time with the lowest heat exposure for a location $\ell \in \mathrm{L}$, one must consider constraints such as the opening hours $\left[\mathrm{t}_{\text {open }}(\ell), \mathrm{t}_{\text {close }}(\ell)\right]$ of $\ell$. We use the heat exposure of the optimal path between the starting point $\mathrm{s}$ and location $\ell$ as the objective function to minimize (see section 3 - Finding a Route with Minimal Heat Exposure). Finding the time with the minimal heat exposure means to minimize the following objective function $\mathrm{h}(\ell, \mathrm{t})$ :

$$
\mathrm{h}(\ell, \mathrm{t})=\mathrm{w}_{\mathrm{h}}\left(\mathrm{p}^{*}, \mathrm{t}\right)=\min _{\mathrm{p} \in \mathrm{P}_{s \ell}} \mathrm{w}_{\mathrm{h}}(\mathrm{p}, \mathrm{t})=\min _{\mathrm{p} \in \mathrm{P}_{\mathrm{s} \ell}} \sum_{\left(\mathrm{e}, \mathrm{t}^{\prime}\right) \in \mathrm{p}} \mathrm{w}_{\mathrm{h}}\left(\mathrm{e}, \mathrm{t}^{\prime}\right)
$$

where $\mathrm{P}_{\mathrm{s} \ell}$ is the set of all possible paths from $\mathrm{s}$ to $\ell, \mathrm{w}_{\mathrm{h}}(\mathrm{p}, \mathrm{t})$ is the accumulated edge weight of all edges in $\mathrm{p}$ at starting time $\mathrm{t}$, and $\mathrm{w}_{\mathrm{h}}$ is the edge weighting function from equation (2).

Using the objective function defined in equation (5), one can formulate the problem to find a time with minimal heat exposure as an optimization problem with constraints:

$$
\min _{\mathrm{t} \in \mathrm{T}} \mathrm{h}(\ell, \mathrm{t})
$$


s.t. $\quad \mathrm{t} \geq \mathrm{t}_{\text {open }}(\ell)-\mathrm{t}_{\text {walk }}(\ell, \mathrm{t})$

$$
\begin{aligned}
& \mathrm{t} \leq \mathrm{t}_{\text {close }}(\ell)-\left(\mathrm{t}_{\text {walk }}(\ell, \mathrm{t})+\mathrm{t}_{\text {buff }}(\ell)\right) \\
& \mathrm{t} \geq \mathrm{t}_{\text {earliest }}
\end{aligned}
$$

$$
\begin{aligned}
& t \leq t_{\text {latest }} \\
& t \geq t_{\text {now }}
\end{aligned}
$$

Note that location $\ell$ is fixed, as the selection of the location with lowest heat exposure is performed later in step 3 . The constraints (7) and (8) ensure that the arrival at the location is within opening hours. One must ensure that the shop can be reached before it closes. Therefore, one must consider the time needed to walk to location $\ell\left(\mathrm{t}_{\mathrm{walk}}(\ell)\right)$ as well as the time needed to perform e.g. the purchase $\left(\mathrm{t}_{\text {buff }}(\ell)\right)$. But it may make sense to start early in the morning, to arrive at location $\ell$ just in time for opening, so one subtracts the walking time from the opening time. The constraints (9) and (10) are the earliest and latest times respectively desired by the user; they are optional. The last constraint, (11), guarantees that the optimal time is in the future. The walking time $t_{\text {walk }}(\ell, t)$ depends on the starting time $t$, because, conditional on the time, a different optimal route could be selected.

\section{Optimization}

To find the optimal time, one needs an optimization method without derivatives, as the objective function $h(\ell, t)$ is not necessarily derivable. One such method is Brent's method (Brent, 2002), a procedure for the approximation of local optima within an interval $\left[\mathrm{x}_{1}, \mathrm{x}_{2}\right]$.

To apply Brent's method, one has to transform constraints (7)-(11) to the lower and upper limits of an interval. Constraints (7), (9) and (11) can be easily converted to a lower limit, as follows:

$$
\mathrm{t}_{\text {lower }}(\ell, \mathrm{t})=\max \left\{\mathrm{t}_{\text {open }}(\ell)-\mathrm{t}_{\text {walk }}(\ell, \mathrm{t}), \mathrm{t}_{\text {now }}, \mathrm{t}_{\text {earliest }}\right\}
$$

Similarly, we can transform constraints (8) and (10) to an upper limit:

$$
\mathrm{t}_{\text {upper }}(\ell, \mathrm{t})=\min \left\{\mathrm{t}_{\text {close }}(\ell)-\left(\mathrm{t}_{\mathrm{walk}}(\ell, \mathrm{t})+\mathrm{t}_{\text {buff }}(\ell)\right), \mathrm{t}_{\text {latest }}\right\}
$$

It is easy to recognize that the interval $\left[\mathrm{t}_{\text {lower }}(\ell, \mathrm{t}), \mathrm{t}_{\text {upper }}(\ell, \mathrm{t})\right]$ preserves the constraints from the optimization problem defined in equations (7) to (11) above. 
However, Brent's method still cannot be applied, since the lower and upper limits of the interval depend on the starting time $t$ and are therefore not static, as required for Brent's method. To avoid this problem, we propose the introduction of a penalty term and thus the following new objective function $\mathrm{h}^{\prime}(\mathrm{t}, \ell)$ :

$$
\mathrm{h}^{\prime}(\mathrm{t}, \ell)= \begin{cases}\mathrm{h}(\mathrm{t}, \ell) & \text { if } \mathrm{t} \in\left[\mathrm{t}_{\text {lower }}(\ell, \mathrm{t}), \mathrm{t}_{\text {upper }}(\ell, \mathrm{t})\right] \\ \mathrm{h}(\mathrm{t}, \ell)+\mathrm{c} & \text { otherwise }\end{cases}
$$

where $\mathrm{c}$ is a large constant such that $\mathrm{h}(\mathrm{t}, \ell)+\mathrm{c}$ is never selected as the optimal solution if the constraints are violated. Now, we can use the walking time $t_{\text {walk }}^{\text {shortest }}(\ell)$ for the shortest route for the lower and upper limits, which are independent of the actual time. Finally, we can formulate the optimization problem for Brent's method as follows:

$\min _{\mathrm{t} \in \mathrm{T}} \mathrm{h}^{\prime}(\ell, \mathrm{t})$

s.t. $\quad \mathrm{t} \geq \max \left\{\mathrm{t}_{\text {open }}(\ell)-\mathrm{t}_{\text {walk }}^{\text {shortest }}(\ell), \mathrm{t}_{\text {now }}, \mathrm{t}_{\text {earliest }}\right\}$

$$
\mathrm{t} \leq \min \left\{\mathrm{t}_{\text {close }}(\ell)-\left(\mathrm{t}_{\text {walk }}^{\text {shortest }}(\ell)+\mathrm{t}_{\text {buff }}(\ell)\right), \mathrm{t}_{\text {latest }}\right\}
$$

Now we can use Brent's method to find for each location $\ell$ the optimal time $t^{*}$. To avoid the Brent optimizer being trapped in a local optimum, it can be executed several times with different random start points.

\section{Evaluation}

\section{Data}

As map data, we use the data from the OpenStreetMap (OSM) project (OpenStreetMap Foundation, 2016). For weather data, we use the hourly air temperature and relative humidity values originating from the weather station of the German Weather Service (Deutscher Wetterdienste, DWD) in Rheinstetten near Karlsruhe (Deutscher Wetterdienst, 2016). For a finer spatial resolution, we use remote sensing data from a thermal scanner flight provided by the Nachbarschaftsverband Karlsruhe (NVK). The data set consists of two scans, recorded in the morning and in the evening of the 26 September 2008. The data cover an area of $25,805 \mathrm{~m} \times 39,555 \mathrm{~m}(\mathrm{EW} \mathrm{NS})$ and have a resolution of $5,161 \times 7,911$ pixels. The measured surface temperature is in the range of $-1.7^{\circ} \mathrm{C}$ to $+18.3^{\circ} \mathrm{C}$ (morning and evening). The average surface temperatures of the data sets cropped to the evaluated area are $4.18^{\circ} \mathrm{C}$ (morning) and $11.24^{\circ} \mathrm{C}$ (evening). 


\section{Data Preparation}

The OSM data set is cropped to the evaluated area. Afterwards, all ways tagged with highway, railway=platform or public_transport=platform are extracted to obtain the road network.

To compute the edge weights as described in section 0 , we need to make some assumptions. This is because the weather data that we use lack either an appropriate spatial resolution or the required temporal resolution. We therefore assume that the actual spatial variation of the temperature conforms with the spatial variation of the thermal scans (deviation from the mean value). For the relative humidity, we assume a constant value over the study area. We also assume that the temporal variation in the examined area corresponds to the temporal variation measure at the weather station. We apply the morning scan to timestamps between 00:00 and 11:59, and the evening scan between 12:00 and 23:59.

We compute the air temperature at time $t \in \mathrm{T}$ for the raster cell $\mathrm{c}_{\mathrm{ij}}$ as follows:

$$
\mathrm{T}_{\mathrm{a}}\left(\mathrm{t}, \mathrm{c}_{\mathrm{ij}}\right)= \begin{cases}\mathrm{T}_{\mathrm{a}}^{\text {station }}(\mathrm{t})+\delta_{\mathrm{ij}}^{\text {morning }} & \text { if } 0 \leq \mathrm{t}<12, \\ \mathrm{~T}_{\mathrm{a}}^{\text {station }}(\mathrm{t})+\delta_{\mathrm{ij}}^{\text {evening }} & \text { if } 12 \leq \mathrm{t}<24,\end{cases}
$$

where $\mathrm{T}_{\mathrm{a}}^{\text {station }}(\mathrm{t})$ is the air temperature measured at the weather station at time $\mathrm{t}$ and $\delta_{\mathrm{ij}}^{\text {morning }}$ and $\delta_{\mathrm{ij}}^{\text {evening }}$ are the deviations of the raster cell $\mathrm{c}_{\mathrm{ij}}$ from the mean of all raster cells from the morning and evening scans.

We compute an approximation of Steadman's heat index as proposed by Stull (2011, p. 77). Since the heat index is defined for an air temperature between $20^{\circ} \mathrm{C}$ and $50^{\circ} \mathrm{C}$, the air temperature is used as a fall-back value. If the air temperature in a raster cell drops below a comfort threshold $\mathrm{T}_{\mathrm{a}}^{\text {comfort }}$ or $\mathrm{T}_{\mathrm{HI}}^{\text {comfort }}$, that comfort value will be used, because temperatures below this threshold are not considered harmful. It is assumed that a person is walking at a speed of $5 \mathrm{~km} / \mathrm{h}(1.4 \mathrm{~m} / \mathrm{s})$.

For the implementation of the routing, the GraphHopper framework for Java (GraphHopper GmbH, 2016) is used.

To find the optimal point in time, we use the procedure described in section 3.2

For the local search, we use a list of selected OSM tags like shop=supermarket or amenity=pharmacy as search criteria. Only locations for which the opening hours are specified (via the opening_hours tag) and which are within a defined radius around the starting point are considered. We use the direct distance ('as the crow flies'). To reduce the computation effort, a maximum number of results $\mathrm{k}$ can be specified.

The implementation of Brent's method in the Apache Commons Mathematics Library (Apache Software Foundation, 2016) is used with 10 random start points to reduce the risk of finding only a local optimum. 


\section{Results}

\section{Routing}

To evaluate the routing, we selected 1,000 random pairs of start and destination points from the examined area and 10 random dates from the period 1 June to 31 August 2015. For each of the start destination pairs and dates, we performed the evaluation at 7:00, 11:00, 15:00, 19:00 and 23:00, so in total we had 50,000 samples. As a benchmark, we computed the shortest path for each sample.

An overview of our results is given in Fehler! Verweisquelle konnte nicht gefunden werden.. In many cases, the heat exposure can be reduced. On average, the heat exposure decreases by $\sim 4.7 \%$, while at the same time the distance increases by at most $5.76 \%$ on average. In some cases, the heat exposure is reduced by up to $25 \%$. The weighted average of the thermal comfort measure is reduced by $\sim 2^{\circ} \mathrm{C}$ on average. There were only slight differences between the air temperature and the heat index as measures for thermal comfort.

In the example given in Figure 2, the heat exposure is reduced by $17.64 \%$ (temperature) and $18.76 \%$ (heatindex), while at the same time the distance increases by only $0.53 \%$.

Table 1: Overview of the routing results. The values are relative to the shortest route.

\begin{tabular}{ccc}
\hline & temperature & beatindex \\
\hline $\begin{array}{c}\text { Reduction of heat exposure (\% of cases) } \\
\text { overall }\end{array}$ & $79.70 \%$ & $80.53 \%$ \\
more than $5 \%$ & $42.72 \%$ & $45.11 \%$ \\
more than $10 \%$ & $13.81 \%$ & $16.07 \%$ \\
Reduction of heat exposure & & \\
average & $4.63 \%$ & $2.69 \%$ \\
maximum & $25.97 \%$ & \\
average & & $5.76 \%$ \\
Increase of distance & $5.59 \%$ & $2.32{ }^{\circ} \mathrm{C}$ \\
Reduction of relative heat exposure $\left(w_{h} / w_{d}\right)$ & & \\
average & $2.12^{\circ} \mathrm{C}$ & \\
\end{tabular}



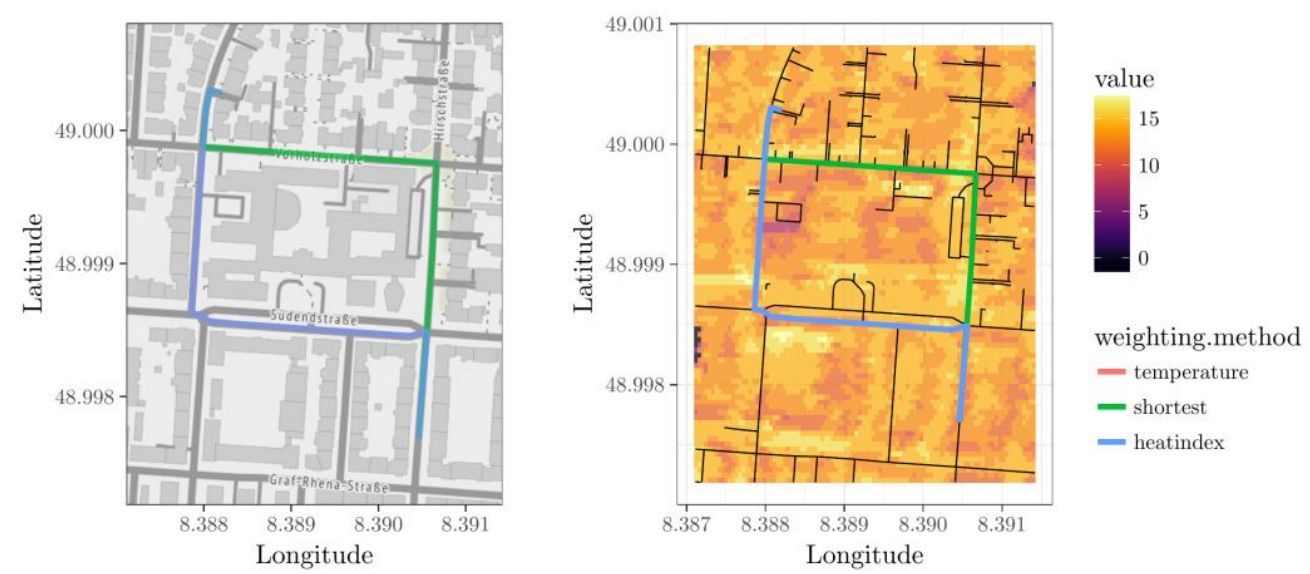

Figure 2: Routing example: both the temperature and the heatindex weighting found the same route. (Map tiles by Stamen Design (2017), under CC BY 3.01. Map data by OSMF (2016), under ODbL22)

\section{Optimal Time}

In order to evaluate the procedure for finding the optimal time, we selected 750 random start points. One of the following three search criteria was then assigned to each of the start points at random: supermarket, bakery, pharmacy. For each of the start points, a random start time $t_{n o w}$ was selected from the period 8:00 to 20:00. The radius was set to750m for all start points, and the maximum number of results was set to 5 . Additionally, for all start points a time buffer $t_{\text {buff }}$ of 15 minutes was assumed.

Table 2: Overview of the results of the combined approach, each compared with the reference solution.

\begin{tabular}{ccc}
\hline & temperature & heatindex \\
\hline $\begin{array}{c}\text { Reduction of heat exposure } \\
\text { \% of cases }\end{array}$ & $68.43 \%$ & $71.08 \%$ \\
Reduction of heat exposure & & \\
Average & $8.09 \%$ & $7.73 \%$ \\
Maximum & $62.2 \%$ & $62.88 \%$ \\
Increase of distance & & \\
Average & $4.60 \%$ & $4.72 \%$ \\
\hline
\end{tabular}

Table 1: Overview of the results of the combined approach, each compared with the reference solution.

\footnotetext{
${ }^{1}$ http://creativecommons.org/licenses/by/3.0

${ }^{2}$ http://www.openstreetmap.org/copyright
} 


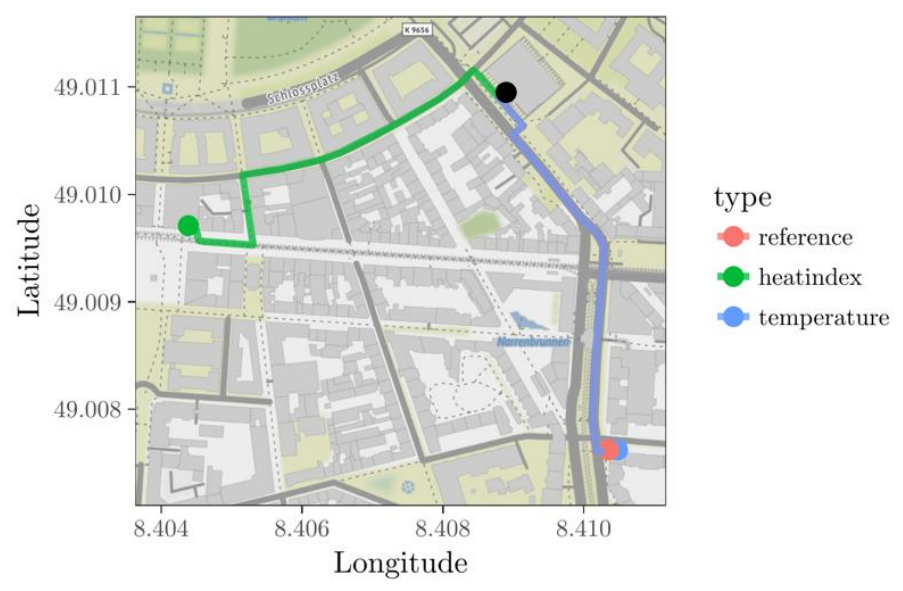

Figure 3: Example for local search, showing the starting point (black dot) as well as the locations ranked best according to the method implemented for each. (Map tiles by Stamen Design (2017), under CC BY 3.0. Map data by OSMF (2016), under ODbL2)

As a reference solution, we used the closest location found during the local search, computed the shortest path from the starting point to this location, and evaluated the heat exposure at time $t_{\text {now. }}$.

The results for the combined approach are given in Table 1. Here the average reduction for exposure to heat compared to the routing approach is significantly higher. This is expected, as heat exposure can vary significantly according to the time of day.

In the example given in Figure 3, the temperature weighting selected the same pharmacy and optimal point in time (9:27) as the reference solution. The heatindex weighting, however, selected a different pharmacy, $476.6 \mathrm{~m}$ instead $434.5 \mathrm{~m}$ away from the start. Furthermore, the method found a different optimal time (19:39) and thus the heat exposure was reduced by $18.49 \%$.

\section{Conclusion}

In this study, we proposed a two-step approach to reduce heat stress for individuals. We achieved this goal by creating a decision support system that computes heat-optimal paths to locations as well the optimal points in time to perform a desired action. We evaluated the method on typical every-day activities such as grocery shopping. We showed that our approach reduces heat stress in the vast majority of cases. This approach is applicable for cities or areas where walking or cycling are valid alternatives. On average, heat stress can be reduced by $\sim 4.7 \%$, while the trade-off in terms of additional distance is quite low (less than $5.8 \%$ on average). We achieved these results, contrary to existing work, for relatively short distances, which average around $2 \mathrm{~km}$. The impact of the results is simple, but significant. By using our approach one can decide for oneself whether the trade-off between additional distance and heat stress reduction is acceptable. Thanks to the very small assumptions made 
in the data set, one can apply the approach quite easily to other cities where basic amenities and services are within walking distances. But these assumptions are also the main restriction of this work. Given the rise of smart cities and (hopefully) the availability of more data sources, the approach could be improved with more fine-detailed data. Even more interesting would be the inclusion of intra-urban temperature forecasts. By incorporating exact forecasts of future values along possible pathways, the optimal point in time as well as the reduction of the heat stress could be improved. Additionally, the inclusion of more complex heat indices could increase the validity for any potential user. These could include the mode of movement (e.g. walking, jogging, cycling) as well as additional environmental factors like the wind or the heat radiated by the sun. Finally, the computation of an overall route which covers a multitude of potential points of interest would be an interesting extension. This could be used for tourists. Here, not only the time and the route with minimal heat exposure should be considered but also the sequence of the locations.

\section{Acknowledgements}

This work is part of the research project BigGIS (reference number: 01IS14012) funded by the Federal Ministry of Education and Research (BMBF) within the frame of the programme 'Management and Analysis of Big Data' in 'ICT 2020 - Research for Innovations'. We thank the Nachbarschaftsverband Karlsruhe for the data from the thermal flights over Karlsruhe.

\section{References}

Apache Software Foundation. (2016). Commons Math: The Apache Commons Mathematics Library. Retrieved from http://commons.apache.org/proper/commons-math/

Basu, R. (2009). High ambient temperature and mortality: a review of epidemiologic studies from 2001 to 2008. Environmental Health, 8, 40. doi:10.1186/1476-069X-8-40

Brent, R. P. (2002). Algorithms for minimization without derivatives. Mineola, NY: Dover Publ.

Dang, C., Iwai, M., Tobe, Y., Umeda, K., \& Sezaki, K. (2013). A framework for pedestrian comfort navigation using multi-modal environmental sensors. Pervasive and Mobile Computing, 9, 421436. doi:10.1016/j.pmcj.2013.01.002

Davis, S. L., Wilson, T. E., White, A. T., \& Frohman, E. M. (2010). Thermoregulation in multiple sclerosis. Journal of Applied Physiology, 109, 1531-1537. doi:10.1152/japplphysiol.00460.2010

Delling, D., Sanders, P., Schultes, D., \& Wagner, D. (2009). Engineering Route Planning Algorithms. In J. Lerner, D. Wagner, \& K. A. Zweig (Eds.), Algorithmics of Large and Complex Networks (pp. 117-139). Springer Berlin Heidelberg. doi:10.1007/978-3-642-02094-0_7

Deutscher Wetterdienst. (2016). Aktuelle stündliche Stationsmessung der Lufttemperatur und Luftfeuchte, Qualitätskontrolle noch nicht vollständig durchlaufen. Retrieved from ftp://ftpcdc.dwd.de/pub/CDC/observations_germany/climate/hourly/air_temperature/recent/stundenw erte_TU_04177_akt.zip

Dijkstra, E. W. (1959). A note on two problems in connexion with graphs. Numerische Mathematik, 1, 269-271. doi:10.1007/BF01386390

Fanger, P. O. (1973). Assessment of man's thermal comfort in practice. British Journal of Industrial Medicine, 30, 313-324. doi:10.1136/oem.30.4.313

GraphHopper GmbH. (2016). GraphHopper Routing Engine. Retrieved from https://graphhopper.com/ 
Hasenfratz, D. (2015). Enabling Large-Scale Urban Air Quality Monitoring with Mobile Sensor Nodes. PhD thesis, ETH-Zürich, Zürich. doi:10.3929/ethz-a-010361120

Hübler, M., Klepper, G., \& Peterson, S. (2007). Costs of Climate Change: The Effects of Rising Temperatures on Health and Productivity in Germany. Kiel Working Paper 1321, Kiel Institute for the World Economy, Kiel. Retrieved from https://www.ifw-members.ifwkiel.de/publications/costs-of-climate-change-the-effects-of-rising-temperatures-on-health-andproductivity-1/Costs $\% 20$ of $\% 20$ Climate $\% 20$ Change $\% 2009-2007$.pdf

Jendritzky, G., Bröde, P., Fiala, D., Havenith, G., Weihs, P., Batchvarova, E., \& DeDear, R. (2010). The Universal Thermal Climate Index UTCI. In A. Matzarakis, H. Mayer, \& F.-M. Chmielewski (Ed.), Proceedings of the 7th Conference on Biometeorology, Freiburg, Germany, April 12-14th, 2010, 20, pp. 184-188. Freibug. Retrieved from http://www.mif.uni-

freiburg.de/biomet/bm7/report20.pdf

Jendritzky, G., Staiger, H., Bucher, K., Graetz, A., \& Laschewski, G. (2000). The Perceived

Temperature: The Method of the Deutscher Wetterdienst for the Assessment of Cold Stress and Heat Load for the Human Body. Internet Workshop on Windchill, April 3-7, 2000, Meteorological Service of Canada. Retrieved from http://www.utci.org/isb/documents/perceived_temperature.pdf

Kaufman, D. E., \& Smith, R. L. (1993). Fastest Paths in Time-Dependent Networks for Intelligent Vehicle-Highway Systems Application. I V H S Journal, 1, 1-11. doi:10.1080/10248079308903779

Oke, T. R. (1982). The energetic basis of the urban heat island. Quarterly Journal of the Royal Meteorological Society, 108, 1-24. doi:10.1002/qj.49710845502

OpenStreetMap Foundation. (2016). The OpenStreetMap Project. Retrieved from http://www.openstreetmap.org/

Orda, A., \& Rom, R. (1990). Shortest-path and Minimum-delay Algorithms in Networks with Timedependent Edge-length. Journal of the $\{$ ACM $\}$, 37, 607-625. doi:10.1145/79147.214078

Prashad, L. (2014). Urban Heat Island. In E. G. Njoku (Ed.), Encyclopedia of Remote Sensing (pp. 878-881). Springer New York. doi:10.1007/978-0-387-36699-9_81

Richter, M. (2015). Urban climate change-related effects on extreme heat events in Rostock, Germany. Urban Ecosystems, 1-18. doi:10.1007/s11252-015-0508-y

Sharker, M. H., Karimi, H. A., \& Zgibor, J. C. (2012). Health-optimal Routing in Pedestrian Navigation Services. Proceedings of the First ACM SIGSPATIAL International Workshop on Use of GIS in Public Health (pp. 1-10). New York: ACM. doi:10.1145/2452516.2452518

Staiger, H., Bucher, K., \& Jendritzky, G. (1997). Gefühlte Temperatur: Die physiologisch gerechte Bewertung von Wärmebelastung und Kältestreß beim Aufenthalt im Freien mit der Maßzahl Grad Celsius. Annalen der Meteorologie. 33, pp. 100-107. Offenbach: Deutscher Wetterdienst.

Retrieved from http://www.dwd.de/DE/leistungen/pbfb_verlag_annalen/pdf_einzelbaende/33_pdf.pdf?_blob $=$ publicationFile\&v $=3$

Staiger, H., Laschewski, G., \& Grätz, A. (2012). The perceived temperature - a versatile index for the assessment of the human thermal environment. Part A: scientific basics. International Journal of Biometeorology, 56, 165-176. doi:10.1007/s00484-011-0409-6

Stamen Design. (2017). Retrieved from http://stamen.com/

Steadman, R. G. (1979a). The Assessment of Sultriness. Part I: A Temperature-Humidity Index Based on Human Physiology and Clothing Science. Journal of Applied Meteorology, 18, 861-873. doi:10.1175/1520-0450(1979)018<0861:TAOSPI >2.0.CO;2

Steadman, R. G. (1979b). The Assessment of Sultriness. Part II: Effects of Wind, Extra Radiation and Barometric Pressure on Apparent Temperature. Journal of Applied Meteorology, 18, 874-885. doi:10.1175/1520-0450(1979)018<0874:TAOSPI >2.0.CO;2

Stull, R. (2011). Meteorology for Scientists and Engineers (3 ed.). Vancouver: University of British Columbia. Retrieved from https://www.eoas.ubc.ca/books/Practical_Meteorology/mse3.html 